\title{
Hierarchical aging pathways and signatures of thermodynamic transition in molecular glasses
}

\author{
Shiyu Liu ${ }^{1,2}$, Yao $\mathrm{Yu}^{1,2^{*}}, \mathrm{Lin}^{\mathrm{Liu}}{ }^{2}$ and $\mathrm{Yue} \mathrm{Wu}^{3^{*}}$
}

\begin{abstract}
When a liquid is supercooled, its structural equilibration time $\tau_{\text {eq }}$ increases sharply approaching the glass transition temperature $T_{\mathrm{g}}$, below which it is kinetically arrested in the out of equilibrium glassy state. Upon annealing below $T_{\mathrm{g}}$, such glassy state relaxes toward the equilibrium state. There is growing evidence that such relaxation process is quite complex, influenced by the complexity of the potential energy landscape. Here we report the observation of a hierarchical aging pathway in the process of the glass-to-supercooled liquid transition in various molecular glass forming systems. Differential scanning calorimetry reveals that the glassy state upon annealing below $T_{\mathrm{g}}$ firstly enters a transient metastable supercooled liquid state with slightly higher enthalpies than that of the equilibrium supercooled liquid state when extrapolated to below $T_{\mathrm{g}}$. This observation is also confirmed by structural measurements via Raman scattering. The dynamics of the transient metastable-to-stable supercooled liquid transition exhibits characteristics of thermodynamic transition at spinodal temperature $T_{\mathrm{sp}}$ slightly above $T_{\mathrm{g}}$, leading to anomalous behaviors such as the appearance of a transition-like behavior in the plot of fragility measurements. These observations imply that the free energy landscape of the supercooled liquid develops complexity with qualitative changes approaching $T_{\mathrm{g}}$ and could have strong influence on the process of the glass transition.
\end{abstract}

Keywords: glass transition, hierarchical aging pathways, thermodynamic transition, metastability

\section{INTRODUCTION}

Glass formation by cooling liquids below the melting point with thwarted crystallization is ubiquitous in nature and plays a prominent role in science and technology [1]. Upon supercooling, the viscosity of the supercooled li- quid increases continuously and sharply, typically exceeding 14 orders of magnitude down to the laboratory glass transition temperature $T_{\mathrm{g}}$, below which the system kinetically falls out of thermal equilibrium and forms a glassy solid [1]. The nature of glass and the glass transition remains one of the challenging fundamental problems in physics [1-6]. Studying the glassy state (GS) relaxation toward the equilibrium below $T_{\mathrm{g}}$, called aging, is an important approach for understanding the GS and the glass transition [7]. For instance, theories focusing on the properties of the equilibrium (excluding referencing to the crystalline state) supercooled liquid state (SLS) predict a hidden thermodynamic phase transition from the SLS to an ideal glassy state at $T_{\mathrm{K}}\left(<T_{\mathrm{g}}\right)$ with an associated divergence of the structural relaxation time $\tau_{\alpha}$ (diverging viscosity of SLS) $[3,6,8]$. However, recent experiments of glasses with long aging times and theoretical analysis, including the examination of a 20-million-yearold amber, raised questions on the existence of the predicted $\tau_{\alpha}$ divergence [9-11].

Aging from the GS to the SLS is commonly assumed to exhibit simple relaxation process determined by a single activation energy. This is reflected by the theory on the dependence of $T_{\mathrm{g}}$ on the heating rate $q^{+}$described by an activated process with a constant activation enthalpy $H^{*}$ [12]. This theory predicts a linear relationship between $\ln \left(q^{+}\right)$and $1 / T_{\mathrm{g}}, \ln \left(q^{+}\right)=-H^{*} /\left(R T_{\mathrm{g}}\right)+$ const., where $R$ is the gas constant $[13,14]$. From the slope of the linear relationship, $H^{*}$ can be determined. Furthermore, it is shown that the fragility $m$ of the SLS near $T_{\mathrm{g}}$, an important parameter of the SLS dynamics [15], can be evaluated by $m=H^{*} /\left(R T_{\mathrm{g}} \ln 10\right)$ [13]. Recently, there is growing evidence suggesting that such single stage activated relaxation scenario cannot capture the complexity

\footnotetext{
${ }^{1}$ School of Materials Science and Engineering and State Key Lab for Materials Processing and Die and Mold Technology, Huazhong University of Science and Technology, Wuhan 430074, China

${ }^{2}$ Wuhan National High Magnetic Field Center, Huazhong University of Science and Technology, Wuhan 430074, China

${ }^{3}$ Department of Physics and Astronomy, University of North Carolina at Chapel Hill, Chapel Hill, NC 27599-3255, USA

* Corresponding authors (emails: ensiyu@mail.hust.edu.cn (Yu Y); yuewu@physics.unc.edu (Wu Y))
} 
of the aging process both from thermodynamic and dynamic point of view. It was found that the volume recovery process of polycarbonate undergoes qualitative change after long aging time $\left(10^{7} \mathrm{~s}\right)$ [16]. Complex aging behaviors such as two-stage relaxation were also found in metallic glass systems [17]. Hierarchical aging pathways and fragile-to-strong transition were observed in $\mathrm{Au}$ based metallic glass systems [18]. These complex aging behaviors point at the important role of the complexity of the potential energy landscape (PEL) where various dominant local minima control the various stages of the aging process [19].

Despite the influence of PEL, another important effect on the aging behaviors originates from the free energy landscape (FEL) which is the landscape of the thermodynamic function reflecting the combined role of enthalpy and entropy. Recent study shows that the FEL is much rougher than that is previously assumed and associates the glass dynamics with fractal change of free energy $[8,20]$. Further studies are needed to explore the effect of FEL on aging. In this work, we report the observation of a complex aging process in various molecular glasses. Systematic calorimetry and Raman spectroscopy investigations reveal that the GS, after annealing below (but near) $T_{\mathrm{g}}$, firstly enters into a transient metastable supercooled liquid state (TMSLS). Here, metastable is with respect to the conventional SLS. Temperature dependences of enthalpy and structural changes and the temperature dependence of the transition rate from TMSLS to SLS all indicate the existence of a spinodal temperature $T_{\mathrm{sp}}$ which is only slightly above $T_{\mathrm{g}}$. These results demonstrate that upon cooling the FEL of the SLS develops complexity with qualitative changes approaching $T_{\mathrm{g}}$. Such complexity of the FEL could play an important role in the process of the laboratory glass transition.

\section{EXPERIMENTAL SECTION}

Six molecular systems, o-terphenyl (OTP, Aladdin 99.0\%), 5-hydroxytryptamine (serotonin, Aladdin 98.0\%), glycerol (Aladdin 99.5\%), ketoprofen (Aladdin 98.0\%), indomethacin (Aladdin 98.0\%), and (S)-(+)-ibuprofen (SIBP, Aladdin 98.5\%) were measured without further purification.

The differential scanning calorimetry (DSC) experiments were carried out in a DSC Q2000 from TA instruments with a liquid nitrogen cooling systems LNCS90. The temperature of DSC system was calibrated by indium with the purity of $99.99 \% .10 \mathrm{mg}$ sample was enclosed in a hermetic aluminum pan under dry nitrogen (at flow rate of $50.00 \mathrm{~mL} \mathrm{~min}^{-1}$ ) to improve the thermal conductivity. The change of thermal lag after calibration was smaller than $0.1 \mathrm{~K}$ with the heating rate below $20.0 \mathrm{~K} \mathrm{~min}^{-1}$.

The fragility measurements of different glass formers were performed by the DSC. The initial GS of each sample was acquired by cooling the sample from $10 \mathrm{~K}$ above its melting temperature to $30 \mathrm{~K}$ below $T_{\mathrm{K}}$ at a constant cooling rate $q^{-}=15.0 \mathrm{~K} \mathrm{~min}^{-1}$. Then the initial GS was reheated directly to above its melting temperature at a constant heating rate $q^{+}$and the $T_{\mathrm{g}}^{\text {onset }}$ was obtained from the heating curve of $C_{\mathrm{P}, \text { norm }}(T)$ as shown in Fig. 1.

The normalized isobaric heat capacity $C_{\mathrm{P}, \text { norm }}(T)$ curves can be obtained from the isobaric heat capacity $C_{\mathrm{P}}(T)$ curves by the following equation:

$C_{\mathrm{P}, \text { norm }}(T)=\frac{C_{\mathrm{P}}(T)-C_{\mathrm{P}, \mathrm{GS}}(T)}{C_{\mathrm{P}, \mathrm{SLS}}(T)-C_{\mathrm{P}, \mathrm{GS}}(T)}$,

where $C_{\mathrm{P}, \mathrm{GS}}$ is the heat capacity of the glassy state and $C_{\mathrm{P}, \text { SLS }}$ is the heat capacity of the supercooled liquid [21].

The numerical calculations of $C_{\mathrm{P}, \text { norm }}(T)$ is based on the Tool-Narayanaswamy-Moynihan (TNM) model. The TNM model is a phenomenological model which can describe the $C_{\mathrm{P}, \text { norm }}(T)$ based on the numerical calculation of relaxation time and fictive temperature $T_{\mathrm{f}}$ on condition that the nonequilibrium behavior of the GS is governed by an activated kinetic process [22-24]. According to the TNM model, the continuous cooling and heating procedure can be considered as a series of small temperature steps and isothermal periods $[12,24]$, during which the relaxation time $\tau$ is mainly determined by the activation energy and fictive temperature $T_{\mathrm{f}}$. After $n$ temperature steps, the fictive temperature $T_{\mathrm{f}, n}$ and the structural relaxation time $\tau_{n}$ can be calculated by the following equation:

$\tau_{n}=\exp \left[\ln (A)+\frac{x \Delta h}{R T_{n}}+\frac{(1-x) \Delta h}{R T_{\mathrm{f}, n}}\right]$,

$T_{\mathrm{f}, n}=T_{0}+\sum_{i=1}^{n} \Delta T_{i}\left\{1-\exp \left[-\left(\sum_{j=\mathrm{i}}^{n} \frac{\Delta T_{j}}{q \tau_{j}}\right)^{\beta_{\mathrm{KWW}}}\right]\right\}$,

where $A$ is a constant, $x$ are fitting parameters relating to the nonlinearity of structural relaxation process, $\Delta h$ is related to the activation energy which is assumed to be unchanged with certain substance, $R$ is the ideal gas constant, $T_{n}$ is the temperature after $n$ temperature steps, $T_{0}$ is the starting temperature well above $T_{\mathrm{g}}$ before cooling, and $q$ is the cooling or heating rate. The normalized heat capacity can be calculated by the differential of fic- 


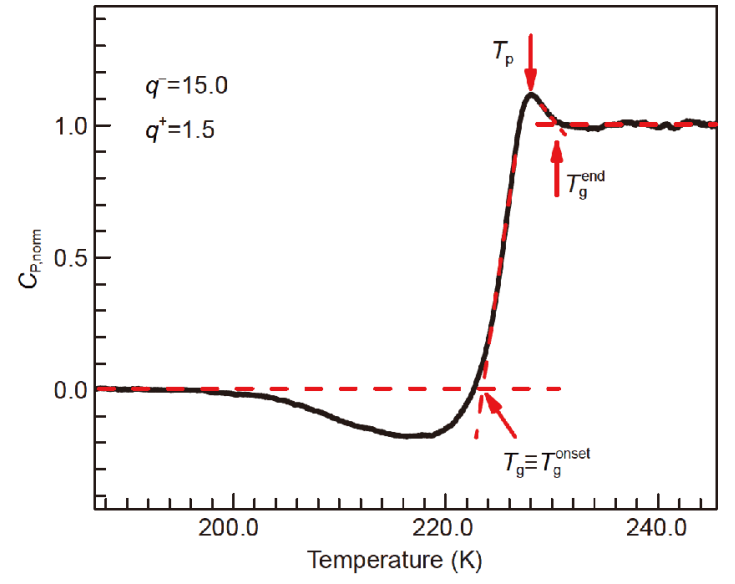

Figure 1 The identification of the characteristic temperatures $T_{\mathrm{g}}^{\text {onset }}, T_{\mathrm{p}}$ and $T_{\mathrm{g}}^{\text {end }} \cdot T_{\mathrm{g}}^{\text {onset }}$ is the onset temperature of glass transition and acquired by the intersection point of the baseline before the glass transition and the tangent line at the inflection point with the maximum slope of the $C_{\mathrm{P}, \text { norm }}(T)$ curve. $T_{\mathrm{p}}$ is at the maximum of the $C_{\mathrm{P}, \text { norm }}(T)$ curve. $T_{\mathrm{g}}^{\text {end }}$ is the intersection point of the baseline and the tangent line at the inflection point after the glass transition.

tive temperature:

$C_{\mathrm{P}, \text { norm }}=\frac{\mathrm{d} T_{\mathrm{f}}}{\mathrm{d} T}=\frac{T_{\mathrm{f}, n}-T_{\mathrm{f}, n-1}}{T_{n}-T_{n-1}}$.

The influences of $q^{-}$and $q^{+}$on the peak height of overshoot of $C_{\mathrm{P}, \text { norm }}(T)$ are discussed with all the other parameters fixed.

Raman spectra were recorded in the $1,550-1,700 \mathrm{~cm}^{-1}$ range by a Horiba JobinYvon LabRAM HR800 Raman spectrometer using the $532 \mathrm{~nm}$ line of a mixed Nd:YAG laser equipped with 1,800 grooves $/ \mathrm{mm}$ gratings. The spectrometer was equipped with a coupled device detector with liquid nitrogen cooled dewar. Temperature of the sample was controlled by a Linkam T95-PE temperature controller which kept temperature fluctuations within $0.10 \mathrm{~K}$. Raman shift was calibrated by monocrystalline silicon before experiment.

\section{RESULTS AND DISCUSSION}

\section{Fragility measurement}

The kinetic behavior of the glass transition was investigated using DSC. Here, the standard fragility measurements of OTP, one of the most widely studied glass formers, indeed seemingly follows the anticipated linear behavior of $\ln \left(q^{+}\right)$vs. 1000/ $T_{\mathrm{g}}^{\text {onset }}$ (Fig. 2a inset). The measured fragility $m=72$ is identical to the previously reported value [25]. However, to our great surprise, when we substantially increased the density of the observation points in $q^{+}$, a transition-like behavior emerges in the $\ln \left(q^{+}\right) v s .1000 / T_{\mathrm{g}}^{\text {onset }}$ plot of OTP (Fig. 2a). As shown in Fig. 2b-f, similar transition-like behavior is also observed in other molecular glass formers including serotonin, glycerol, (S)-(+)-ibuprofen, ketoprofen, and indomethacin. These results show that high density data points are needed to reveal whether such transition-like behavior are also present in other small molecule systems [26].

\section{Heat capacity measurement}

To study the nature of this transition-like behavior, SIBP is selected for further systematic investigations for the following reasons. Its melting point of $324.2 \mathrm{~K}$ is low and presents no thermal stability issue for organic molecules. The SLS of SIBP is extraordinarily stable against crystallization, persisting at least for days over the entire supercooled liquid region (Fig. S1), opening the window for a systematic investigation of the transition-like behavior. In the stable crystalline phase of SIBP, all molecules are in the form of cyclic dimers via intermolecular hydrogen bonding (Fig. S2) [27]. The liquid phase consists of monomers and multimers via intermolecular hydrogen bonding, including dimers, trimers and tetramers where the cyclic dimer is the most energetically favorable structure with the strongest intermolecular hydrogen bonding [28]. Here, the change of the strength and the population of hydrogen bondings provide a sensitive measure for detecting the change of the liquid structure.

The calorimetry signature of the glass transition upon heating is the sharp increase of the normalized isobaric specific heat $C_{\mathrm{P} \text {,norm }}(T)$ and emergence of an overshoot around $T_{\mathrm{g}}$ under the employed heating rate. This overshoot is due to a rapid recovery of the lost enthalpy through the relaxation process [12]. The upper panel of Fig. 3a shows a set of representative $C_{\mathrm{P} \text {,norm }}(T)$ curves for $q^{+}$ranging from 1.0 to $15.0 \mathrm{~K} \mathrm{~min}^{-1}$. The initial GS is prepared by cooling the liquid from $333.2 \mathrm{~K}$ down to $193.2 \mathrm{~K}$ under the constant cooling rate $q^{-}=$ $15.0 \mathrm{~K} \mathrm{~min}^{-1}$. It is very interesting to note that the peak height at $T_{\mathrm{p}}$ of the $C_{\mathrm{P} \text {,norm }}(T)$ overshoot firstly decreases with increasing $q^{+}$in the low $-q^{+}$range, reaching a minimum at $T=230.6 \mathrm{~K}$ under $q^{+}=3.0 \mathrm{~K} \mathrm{~min}^{-1}$. In contrast, the peak height at $T_{\mathrm{p}}$ of the $C_{\mathrm{P}, \text { norm }}(T)$ overshoot increases with increasing $q^{+}$in the high- $q^{+}$range above $q^{+}$ $=3.0 \mathrm{~K} \mathrm{~min}^{-1}$. The passage time $\tau_{\text {avg }} \equiv\left(T_{\mathrm{g}}^{\text {end }}-T_{\mathrm{g}}^{\text {onset }}\right) / q^{+}$ from the GS to the SLS reflects clearly this abrupt change [15]. The lower panel of Fig. 3a plots $\tau_{\text {avg }} v s .1000 / T_{\mathrm{p}}$ by varying $q^{+}$from 1.0 to $20.0 \mathrm{~K} \mathrm{~min}^{-1}$ and the abrupt 

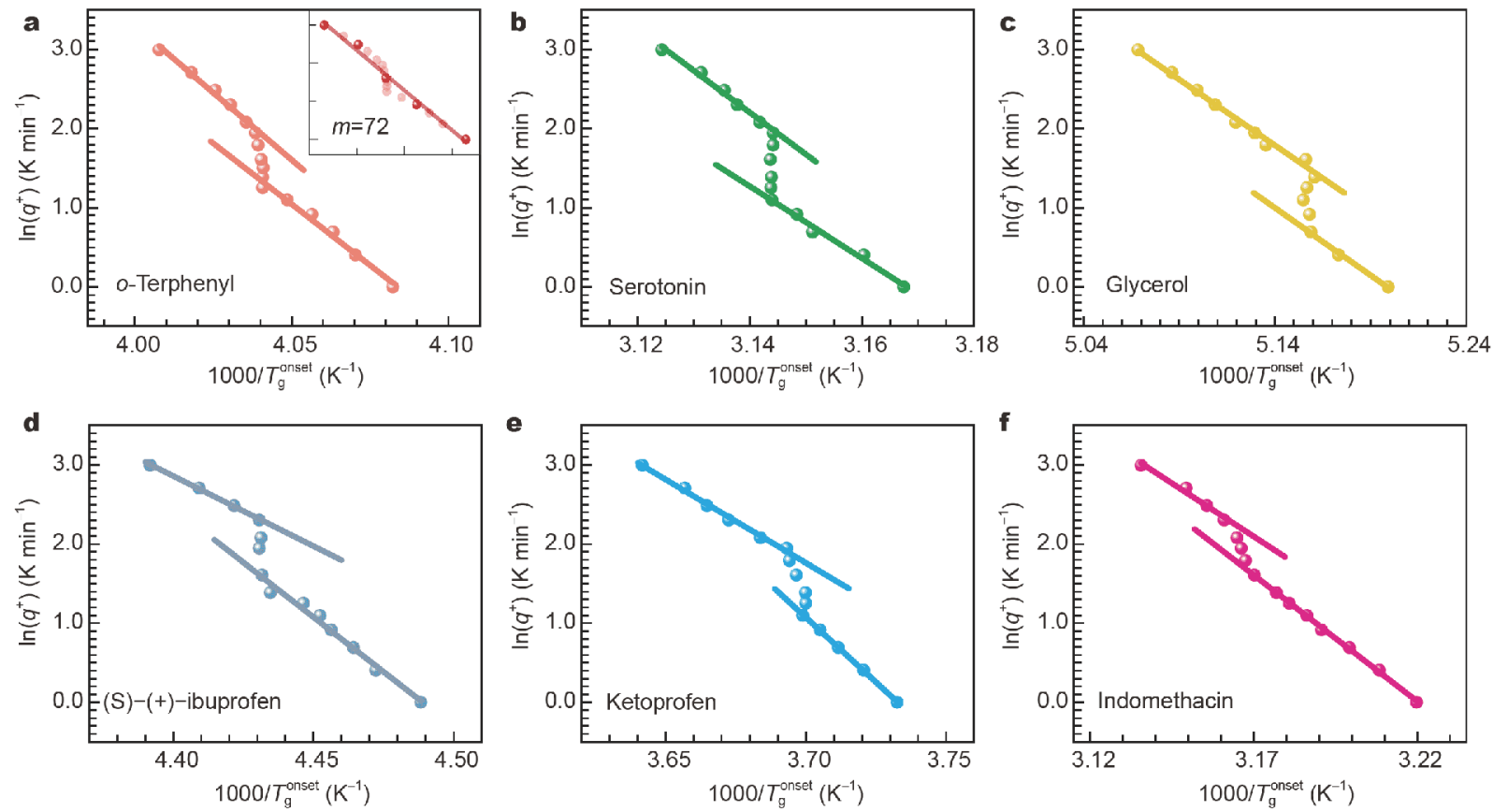

Figure 2 Calorimetry measurements of glass transition in molecular liquids. (a) Plot of $\ln \left(q^{+}\right) v s .1000 / T_{\mathrm{g}}^{\text {onset }}$ acquired by sixteen observation points for OTP. Plot of $\ln \left(q^{+}\right) v s .1000 / T_{\mathrm{g}}^{\text {onset }}$ acquired by five observation points are shown in the inset with the sixteen observation points shown as faded background. Plots of $\ln \left(q^{+}\right) v s .1000 / T_{\mathrm{g}}^{\text {onset }}$ acquired by sixteen observation points for serotonin (b), glycerol (c), (S) $-(+)-$ ibuprofen (d), ketoprofen (e), and indomethacin (f).

change of $\tau_{\text {avg }}$ occurs at $T_{\mathrm{p}}=230.6 \mathrm{~K}$. These experiments are also repeated with different initial GS, including using $q^{-}=0.5 \mathrm{~K} \mathrm{~min}^{-1}$ instead of $q^{-}=15.0 \mathrm{~K} \mathrm{~min}^{-1}$ used in the above experiments (Fig. 3b). As the overshoot of $C_{\mathrm{P}}$ curve is closely related to the kinetics of the relaxation process, the abnormal changes of the peak height of the overshoot are directly related to the abnormal behavior of $\tau_{\text {avg }}$ versus $T$, where a rapid decrease of $\tau_{\text {avg }}$, far exceeding that of an activated behavior, is observed as $T$ increases approaching $T_{\mathrm{p}}=230.6 \mathrm{~K}$.

Also evaluated is the case of $q^{-}=q^{+}$where each $C_{\mathrm{P}, \text { norm }}(T)$ curve is obtained with the heating rate $q^{+}$equal to the cooling rate $q^{-}$under which the initial GS is prepared (Fig. 3c) [12]. In all these cases, there is always an overshoot minimum occurring at the same temperature of $T=230.6 \pm 0.3 \mathrm{~K}$ and an abrupt change of $\tau_{\text {avg }}$ versus $1000 / T_{\mathrm{p}}$ at the same $T_{\mathrm{p}}$. These results indicate that the equilibration time $\tau_{\text {eq }}$ decreases abruptly at $T=230.6 \pm$ $0.3 \mathrm{~K}$, denoted as $T_{\mathrm{sp}}$, which is independent of the employed cooling/heating rates. The above mentioned $C_{\mathrm{P}, \text { norm }}(T)$ are very different from what are expected by the conventional activated kinetic process [29]. In such theory, the conventional $C_{\mathrm{P} \text {,norm }}(T)$ behavior can be cal- culated numerically based on the TNM model, which describes the nonequilibrium behavior of the GS governed by a single stage activated kinetic process $[13,22,23,30]$. Fig. $3 d-f$ show the corresponding calculated $C_{\mathrm{P}, \text { norm }}(T)$ curves and the $\tau_{\text {avg }}$ versus $1000 / T_{\mathrm{p}}$ under various conditions. Here, the overshoot minimum and the abrupt change of $\tau_{\text {avg }}$ are completely absent. Moreover, it is of note that the overshoot minimum and the abrupt change of $\tau_{\text {avg }}$ should be absent even considering dynamic heterogeneity [31]. These results are characteristic behaviors of a purely kinetic process with no abrupt change of activation energies over the involved temperature range. Clearly, the relaxation process cannot be described by the simple process with constant activation enthalpy.

\section{Enthalpy measurement}

Since the observed abnormal dynamic behaviors cannot be explained qualitatively by a purely kinetic process, possible thermodynamic origin was investigated. Here, the GS prepared under $q^{-}=15.0 \mathrm{~K} \mathrm{~min}^{-1}$ down to $193.2 \mathrm{~K}$ was heated with $q^{+}=15.0 \mathrm{~K} \mathrm{~min}^{-1}$ from $193.2 \mathrm{~K}$ to a chosen $T$ and held there isothermally for a time period of 

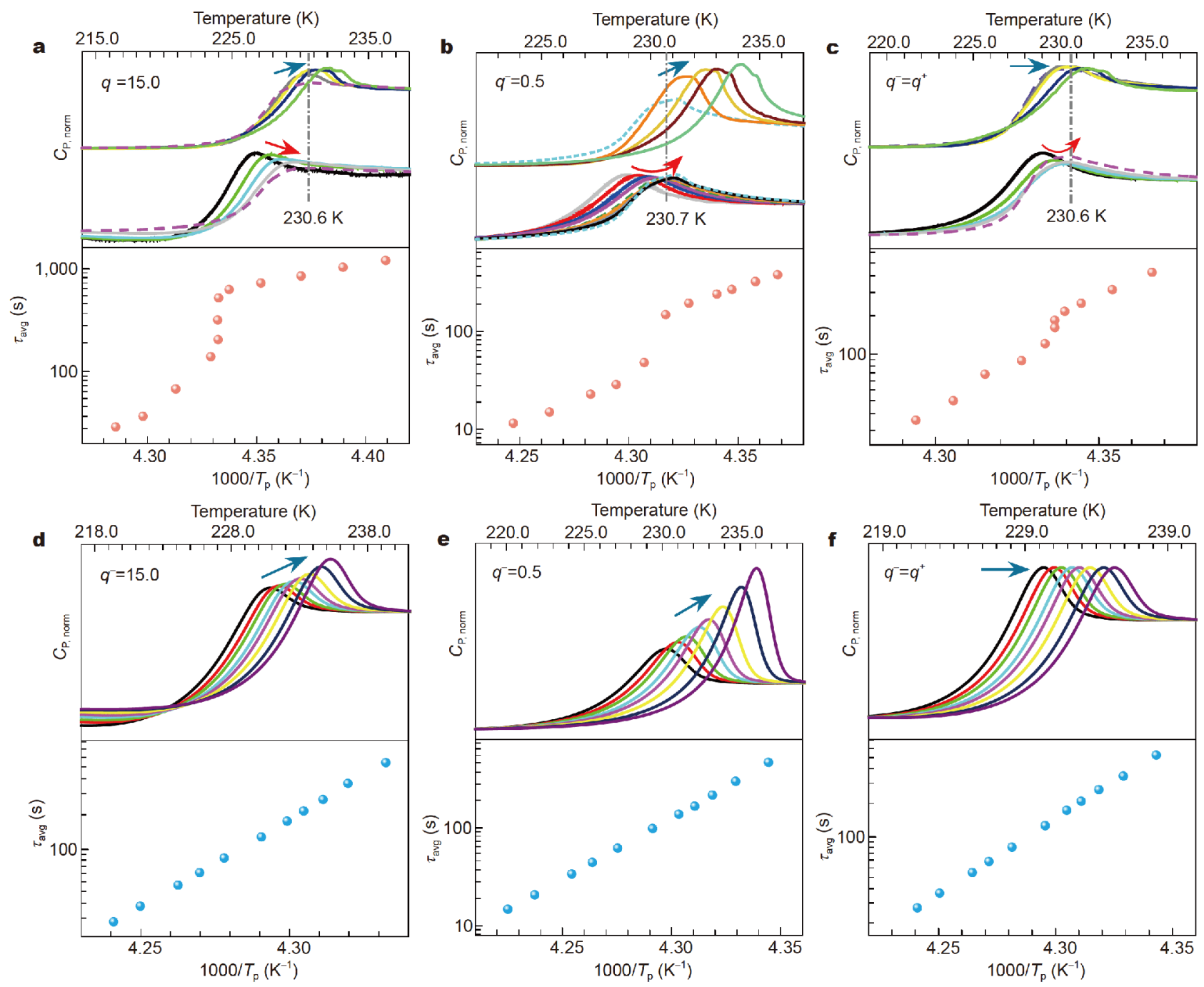

Figure 3 Calorimetry measurements of SIBP glass transition and numerical calculations. (a) The set of $C_{\mathrm{P}, \text { norm }}(T)$ curves with $q^{+}$from 1.0 to $15.0 \mathrm{~K} \mathrm{~min}^{-1}$ with the initial GSs prepared under cooling rate $q^{-}=15.0 \mathrm{~K} \mathrm{~min}^{-1}$ (upper panel). The corresponding $\tau_{\text {avg }}$ versus $1000 / T_{\mathrm{p}}$ (lower panel). (b) The set of $C_{\mathrm{P}, \text { norm }}(T)$ curves with $q^{+}$from 1.0 to $15.0 \mathrm{~K} \mathrm{~min}^{-1}$ with the initial GS prepared under the cooling rate $q^{-}=0.5 \mathrm{~K}$ min ${ }^{-1}$ (upper panel). The corresponding $\tau_{\text {avg }}$ versus $1000 / T_{\mathrm{p}}$ (lower panel). (c) The set of $C_{\mathrm{P}, \mathrm{norm}}(T)$ curves with $q^{+}$from 1.0 to $15.0 \mathrm{~K} \mathrm{~min}{ }^{-1}$ with the initial GSs prepared under cooling rate $q^{-}=q^{+}$(upper panel). The corresponding $\tau_{\text {avg }}$ versus $1000 / T_{\mathrm{p}}$ (lower panel). Also shown are the corresponding results from numerical calculations with the initial GSs prepared under cooling rate $q^{-}=15.0 \mathrm{~K} \mathrm{~min}^{-1}$ (d), $q^{-}=0.5 \mathrm{~K} \mathrm{~min}^{-1}$ (e), and $q^{-}=q^{+}$(f).

$t_{\text {isotherm }}$ (Fig. S3); the enthalpy $H_{\text {isotherm }}(T)$ was obtained by integrating the heat flow over the entire heating and isothermal time period (Fig. S3). This experiment is possible because of the extraordinary stability of SIBP against crystallization.

Fig. 4a shows the measured $H_{\text {isotherm }}(T)$ with $t_{\text {isotherm }}=$ 3,600 s. Above $230.2 \mathrm{~K}, H_{\text {isotherm }}(T)$ shows a linear temperature dependence as expected from a SLS [32]. Interestingly, from 223.2 to $227.2 \mathrm{~K}, H_{\text {isotherm }}(T)$ also shows a linear temperature dependence with a comparable slope as $H_{\text {isotherm }}(T)$ above $230.2 \mathrm{~K}$ but slightly shifted upward.
Further, the features of $H_{\text {isotherm }}(T)$ above $223.2 \mathrm{~K}$ change very little with $t_{\text {isotherm varying from } 1,200 \text { to } 3,600 \mathrm{~s}}$ (Fig. 4b). This is very different from the typical GS where $H_{\text {isotherm }}(T)$ changes continuously with increasing $t_{\text {isotherm }}$. Above results suggest that the system firstly enters a transient metastable state in the temperature range from 223.2 to $227.2 \mathrm{~K}$. Here, the metastability is referred to the SLS which is itself metastable with respect to the crystalline state. The linearity of the enthalpy versus temperature curve, $H_{\text {isotherm }}(T)$, the independence of this curve on $t_{\text {isotherm }}$ from 1,200 to $3,600 \mathrm{~s}$, and the similar 

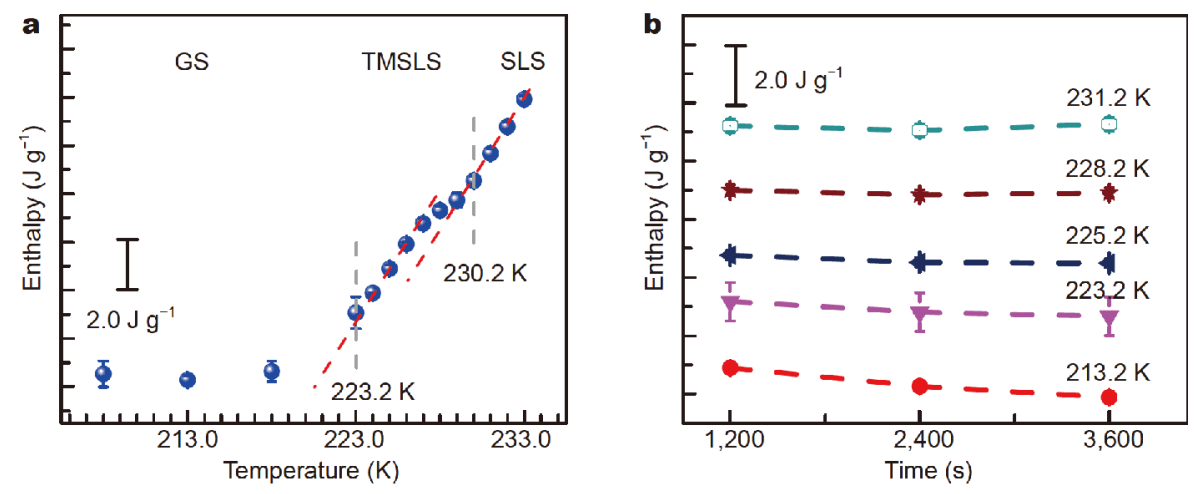

Figure 4 Enthalpy measurements of SIBP glass transition. (a) Enthalpy at $T$ after held isothermally for 3,600 $\mathrm{s}$ represented by $H_{\text {isotherm }}(T)$. (b) $H_{\text {isotherm }}(T)$ acquired with $t_{\text {isotherm }}$ varying from 1,200 to $3,600 \mathrm{~s}$.
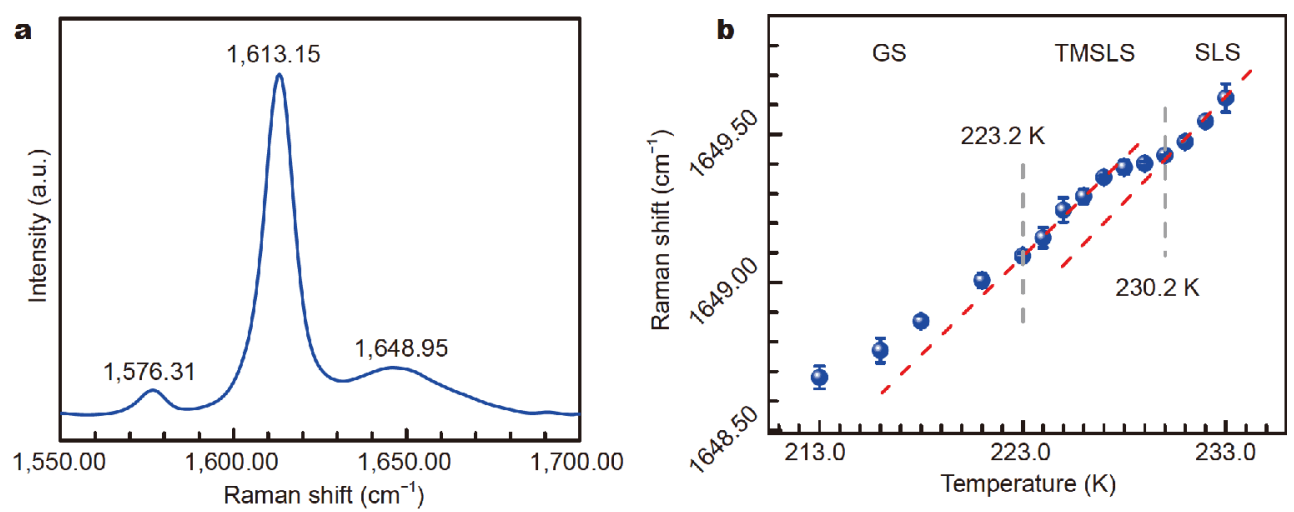

Figure 5 Raman measurements of SIBP glass transition. (a) A typical Raman spectrum acquired at $223.2 \mathrm{~K}$. (b) The temperature dependence of the Raman shift of the band around $1,649.00 \mathrm{~cm}^{-1}$.

slope of this curve to that of the supercooled liquid state indicate that the system enters a transient metastable state with established thermal equilibrium in the transient metastable state. Thus, the system enters a well-defined liquid (TMSLS) state after 1,200 s annealing and remains there at least for 3,600 $\mathrm{s}$ in the temperature region from 223.2 to $227.2 \mathrm{~K}$ [33]. It is of note that the TMSLS merges to the SLS at $230.2 \pm 0.5 \mathrm{~K}$, identical to $T_{\mathrm{sp}}=230.6 \pm 0.3 \mathrm{~K}$ within experimental uncertainties. Such dynamic and thermodynamic signatures are characteristics of a spinodal transition at $T_{\mathrm{sp}}=230.6 \mathrm{~K}$ where the free energy barrier separating TMSLS and SLS disappears [34].

\section{Raman measurement}

The possible structural origin of the TMSLS to SLS transition was investigated by Raman spectroscopy. Here, the SIBP liquid was firstly cooled from 333.2 to $153.2 \mathrm{~K}$ at a rate of $15.0 \mathrm{~K} \mathrm{~min}^{-1}$, then heated step by step to $235.2 \mathrm{~K}$ with an interval of $5.0 \mathrm{~K}$ in the temperature range of 213.2 to $223.2 \mathrm{~K}$ and an interval of $1.0 \mathrm{~K}$ above $223.2 \mathrm{~K}$. At each step after equilibrating the system for 10 min, ten Raman spectra were taken isothermally with exposure time of $10 \mathrm{~s}$ and accumulation number was 6 . The experiments were repeated three times to eliminate the statistical error. A typical Raman spectrum acquired at $223.2 \mathrm{~K}$ is shown in Fig. 5a. There are three characteristic bands at 1,576.31, $1,613.15$, and $1,648.95 \mathrm{~cm}^{-1}$. Here, the broad band around $1,649.00 \mathrm{~cm}^{-1}$ corresponds to the vibration mode involving the carboxylic $\mathrm{C}=\mathrm{O}$ group $[35,36]$, and an upshift of this Raman band reflects the weakening of the hydrogen bonding [37]. The temperature dependence of this Raman shift is shown in Fig. 5b, exhibiting a similar behavior as that of $H_{\text {isotherm }}(T)$ with the two straight lines merging at $230.2 \pm 0.5 \mathrm{~K}$.

The Raman results reveal that the hydrogen bonding is weaker in the TMSLS than that in the SLS. This is fully consistent with results of the previous study showing the increase of hydrogen bonding population upon cooling in the supercooled liquid [28]. TMSLS corresponds to the state with higher monomer population and insufficient 


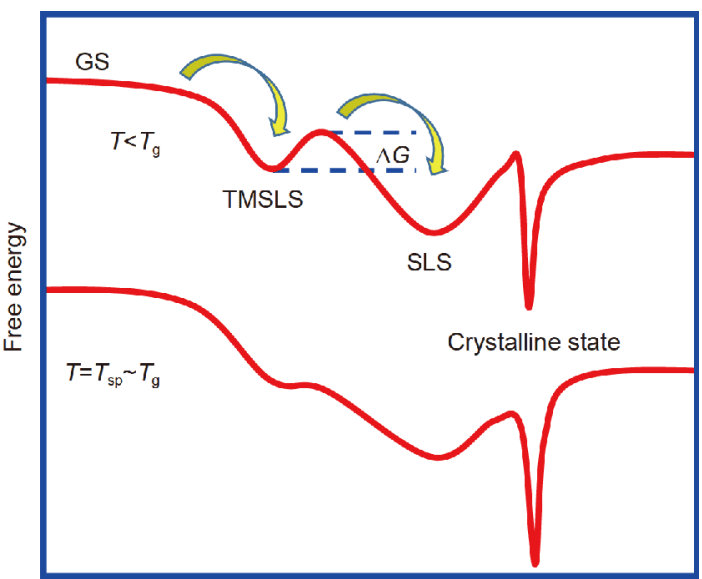

Figure 6 Illustration of the free energy landscape. Upon heating, the GS firstly enters the TMSLS at an intermediate free energy minimum. The transition from TMSLS to SLS speeds up dramatically at $T_{\mathrm{sp}} \sim T_{\mathrm{g}}$ above which the free energy local minimum disappears.

conversion from multimers to cyclic dimers compared with the equilibrium SLS.

\section{Discussion}

What could be the mechanism and the structural basis underlying the emergence of metastable states? In structural glasses where disorders are self-induced, geometrical factors could lead to frustrations [38,39]. Metastable states could arise from such geometrical frustration mechanism depending on the degree of frustration [40]. One of the frustration mechanisms is proposed by Tanaka [41]. Here, the growth of local crystalline bond orientational ordering ( $\mathrm{CBOO}$ ) provides the incompatibility with liquid structure and frustration, leading to energetic and/or entropic frustration and sharp slowdown in dynamics [41]. This scenario can be envisioned in the SLS of SIBP.

Upon cooling, the growth of $\mathrm{CBOO}$ can be identified with the increase of the number of cyclic dimers which are the only building blocks for periodic space filling of SIBP (Fig. S2). Although cyclic dimers are by far the most energetically favorable local configurations, linear dimers are also present with significant population [28], perhaps driven by entropy and interactions such as $\mathrm{CH} / \pi$ bonds [42]. Upon cooling, the energetically driven growth of the number of cyclic dimers $n_{\text {cycdim }}$ has to compete with linear dimers and other configurations which are incompatible with periodic space filling. This could then lead to frustration and the formation of TMSLS in the FEL, $G\left(T, n_{\text {cycdim }}\right)$, where the number of cyclic dimers $n_{\text {cycdim }}$ (TMSLS) is lower than what it should be in the SLS, $n_{\text {cycdim }}$ (SLS), at the given temperature. Such scenario is fully consistent with the Raman results where an increase of hydrogen bond strength is observed in the TMSLS to SLS transition. As the cooling process eventually becomes non-quasistatic, SIBP with increasing $n_{\text {cycdim }}$ firstly enters the TMSLS below $T_{\text {sp }}$, and the emergence of TMSLS causes a significant increase of the equilibration time $\tau_{\text {eq }}$ for establishing the SLS. The emergence and the growth of free energy barriers below $T_{\mathrm{sp}}$ could contribute significantly to the glass transition.

The hierarchical relaxation process reveals the complexity of the FEL below $T_{\mathrm{sp}}$. Upon heating, as illustrated in Fig. 6, the annealed GS firstly enters the TMSLS with closer resemblance in structural order such as $n_{\text {cycdim. }}$. Longer time annealing would eventually lead to structural relaxation to the SLS. The last stage of relaxation becomes much faster when the temperature reaches the spinodal point $T_{\mathrm{sp}}$.

\section{CONCLUSIONS}

This work reports the observation of a two-stage hierarchical aging process in molecular glasses. It reveals that the glassy state, after annealing below $T_{\mathrm{g}}$, firstly enters into a TMSLS. The TMSLS then undergoes a transition to the SLS above $T_{\mathrm{g}}$. Moreover, it is shown that there is a spinodal temperature $T_{\mathrm{sp}}$ associated with the TMSLS-toSLS transition. Here, $T_{\mathrm{sp}}>T_{\mathrm{g}}$ but is found to be close to $T_{\mathrm{g}}$. The transition time $\tau$ from TMSLS to SLS decreases steeply at the spinodal temperature. This steep decrease of $\tau$ is evidenced by several anomalous behaviors. In dynamic properties, an anomalous transition-like behavior is observed in the plot of the fragility measurement, unlike the expected linear behavior of simple aging process; the overshoot in the specific heat $C_{\mathrm{P}} v$ s. $T$ associated with the glass transition exhibits a minimum at a given heating rate corresponding to $T_{\mathrm{sp}}$, unlike the behavior of monotonic increase (or unchanged) as expected from single stage aging process. This is further supported by measurements of enthalpy $v s$. $T$ after aging as well as structural changes $v s$. $T$ after aging, both showing a transition at $T_{\text {sp }}$. These results show that the FEL of the supercooled liquid could develop important complexity with qualitative changes below the spinodal temperature $T_{\mathrm{sp}}$. Such complexity in FEL below $T_{\mathrm{sp}}$ could facilitate the onset of the laboratory glass transition.

\section{Received 3 November 2018; accepted 13 December 2018;} published online 9 January 2019

1 Ediger MD, Angell CA, Nagel SR. Supercooled liquids and glasses. J Phys Chem, 1996, 100: 13200-13212 
2 Angell CA. Formation of glasses from liquids and biopolymers. Science, 1995, 267: 1924-1935

3 Berthier L, Biroli G. Theoretical perspective on the glass transition and amorphous materials. Rev Mod Phys, 2011, 83: 587-645

4 Dyre JC. Colloquium: The glass transition and elastic models of glass-forming liquids. Rev Mod Phys, 2006, 78: 953-972

5 Chandler D, Garrahan JP. Dynamics on the way to forming glass: bubbles in space-time. Annu Rev Phys Chem, 2010, 61: 191-217

6 Cavagna A. Supercooled liquids for pedestrians. Phys Rep, 2009, 476: 51-124

7 Swallen SF, Kearns KL, Mapes MK, et al. Organic glasses with exceptional thermodynamic and kinetic stability. Science, 2007, 315: $353-356$

8 Lubchenko V, Wolynes PG. Theory of structural glasses and supercooled liquids. Annu Rev Phys Chem, 2007, 58: 235-266

9 Hecksher T, Nielsen AI, Olsen NB, et al. Little evidence for dynamic divergences in ultraviscous molecular liquids. Nat Phys, 2008, 4: 737-741

10 Zhao J, Simon SL, McKenna GB. Using 20-million-year-old amber to test the super-Arrhenius behaviour of glass-forming systems. Nat Commun, 2013, 4: 1783

11 Mauro JC, Yue Y, Ellison AJ, et al. Viscosity of glass-forming liquids. Proc Natl Acad Sci USA, 2009, 106: 19780-19784

12 Moynihan CT. Structural relaxation and the glass transition. Rev Mineral Geochem, 1995, 32: 1-19

13 Moynihan CT, Easteal AJ, Wilder J, et al. Dependence of the glass transition temperature on heating and cooling rate. J Phys Chem, 1974, 78: 2673-2677

14 Moynihan CT. Correlation between the width of the glass transition region and the temperature dependence of the viscosity of high- $T_{\mathrm{g}}$ glasses. J Am Ceramic Soc, 1993, 76: 1081-1087

15 Böhmer R, Ngai KL, Angell CA, et al. Nonexponential relaxations in strong and fragile glass formers. J Chem Phys, 1993, 99: 42014209

16 Wimberger-Friedl $\mathrm{R}$, de Bruin JG. The very long-term volume recovery of polycarbonate: is self-retardation finite? Macromolecules, 1996, 29: 4992-4997

17 Luo P, Wen P, Bai HY, et al. Relaxation decoupling in metallic glasses at low temperatures. Phys Rev Lett, 2017, 118: 225901

18 Gallino I, Cangialosi D, Evenson Z, et al. Hierarchical aging pathways and reversible fragile-to-strong transition upon annealing of a metallic glass former. Acta Mater, 2018, 144: 400-410

19 Goldstein M. Viscous liquids and the glass transition: a potential energy barrier picture. J Chem Phys, 1969, 51: 3728-3739

20 Charbonneau P, Kurchan J, Parisi G, et al. Fractal free energy landscapes in structural glasses. Nat Commun, 2014, 5: 3725

21 Kubaschewski O, Alcock CB, Spencer P. Materials Thermochemistry. Oxford: Pergamon Press, 1993

22 Tool AQ. Relation between inelastic deformability and thermal expansion of glass in its annealing range. J Am Ceramic Soc, 1946, 29: $240-253$

23 Narayanaswamy OS. A model of structural relaxation in glass. J Am Ceramic Soc, 1971, 54: 491-498

24 Debolt MA, Easteal AJ, Macedo PB, et al. Analysis of structural relaxation in glass using rate heating data. J Am Ceramic Soc, 1976, 59: $16-21$

25 Crowley KJ, Zografi G. The use of thermal methods for predicting glass-former fragility. ThermoChim Acta, 2001, 380: 79-93

26 Chen Z, Zhao L, Tu W, et al. Dependence of calorimetric glass transition profiles on relaxation dynamics in non-polymeric glass formers. J Non-Crystalline Solids, 2016, 433: 20-27

27 Freer AA, Bunyan JM, Shankland N, et al. Structure of (S)(+)-ibuprofen. Acta Crystlogr C Cryst Struct Commun, 1993, 49: $1378-1380$

28 Bras AR, Noronha JP, Antunes AMM, et al. Molecular motions in amorphous ibuprofen as studied by broadband dielectric spectroscopy. J Phys Chem B, 2008, 112: 11087-11099

29 Badrinarayanan $\mathrm{P}$, Zheng $\mathrm{W}, \mathrm{Li} \mathrm{Q}$, et al. The glass transition temperature versus the fictive temperature. J Non-Crystalline Solids, 2007, 353: 2603-2612

30 Hodge IM, Berens AR. Effects of annealing and prior history on enthalpy relaxation in glassy polymers. 2. Mathematical modeling. Macromolecules, 1982, 15: 762-770

31 Wolynes PG. Spatiotemporal structures in aging and rejuvenating glasses. Proc Natl Acad Sci USA, 2009, 106: 1353-1358

32 Debenedetti PG, Stillinger FH. Supercooled liquids and the glass transition. Nature, 2001, 410: 259-267

33 Palmer RG. Broken ergodicity. Adv Phys, 1982, 31: 669-735

34 Binder K. Nucleation barriers, spinodals, and the Ginzburg criterion. Phys Rev A, 1984, 29: 341-349

35 Vueba ML, Pina ME, Batista de Carvalho LAE. Conformational stability of ibuprofen: Assessed by DFT calculations and optical vibrational spectroscopy. J Pharmaceutical Sci, 2008, 97: 845-859

36 Rossi B, Verrocchio P, Viliani G, et al. Vibrational properties of ibuprofen-cyclodextrin inclusion complexes investigated by Raman scattering and numerical simulation. J Raman Spectrosc, 2009, 40: 453-458

37 Crupi V, Majolino D, Venuti V, et al. Temperature effect on the vibrational dynamics of cyclodextrin inclusion complexes: investigation by FTIR-ATR spectroscopy and numerical simulation. J Phys Chem A, 2010, 114: 6811-6817

38 Nelson DR. Defects and Geometry in Condensed Matter Physics. Cambridge: Cambridge University Press, 2002

39 Kivelson D, Kivelson SA, Zhao X, et al. A thermodynamic theory of supercooled liquids. Phys A-Stat Mech Appl, 1995, 219: 27-38

40 Kivelson D, Tarjus G. Apparent polyamorphism and frustration. J Non-Crystalline Solids, 2002, 307-310: 630-636

41 Tanaka H. Bond orientational order in liquids: Towards a unified description of water-like anomalies, liquid-liquid transition, glass transition, and crystallization. Eur Phys J E, 2012, 35: 113

42 Nishio $\mathrm{M}$. $\mathrm{CH} / \pi$ hydrogen bonds in crystals. CrystEngComm, 2004, 6: $130-158$

Acknowledgements This work was partially supported by the National Basic Research Program of China (2015CB856801). We are grateful to the Analytical and Testing Center, Huazhong University of Science and Technology for technical assistance.

Author contributions Liu S conducted the experiments. All authors contributed to experimental design, data analysis, and interpretation. $\mathrm{Liu} \mathrm{S}, \mathrm{Yu} \mathrm{Y}$, and $\mathrm{Wu} \mathrm{Y}$ wrote the manuscript. Yu Y and $\mathrm{Wu} \mathrm{Y}$ directed the research.

Conflict of interest The authors declare that they have no conflict of interest.

Supplementary information See supplementary material for specific details about the crystalline structure of SIBP, the stability of supercooled liquid, and the method of enthalpy measurement. All the supporting data are available in the online version of the paper. 


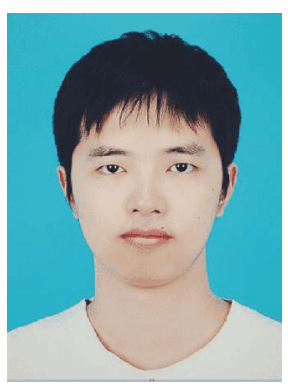

Shiyu Liu received his bachelor's degree from Huazhong University of Science and Technology in 2011 and is pursuing $\mathrm{PhD}$ degree at Huazhong University of Science and Technology. His research interest mainly focuses on the liquid complexity, the nature of glass transition and melting.
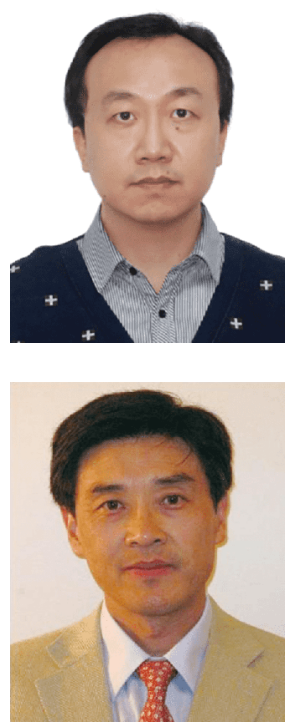

Yao Yu is currently an associated professor in Huazhong University of Science and Technology. His research interest mainly focuses on the properties of liquid and liquid-related transitions, including glass transition, crystallization and melting, and applications of liquid, such as the application of electrolyte for energy storage.
Yue Wu is currently a Kenan distinguished Professor in the University of North Carolina at Chapel Hill. His current research interest includes understanding of the nature of glass transition and novel properties of nanoconfined liquids.

\section{小分子玻璃中的分层老化路径和热力学转变迹象}

刘诗宇 ${ }^{1,2}$, 于尧 ${ }^{1,2^{*}}$, 柳林 ${ }^{2}$, 吴跃 $3^{3^{*}}$

摘要 当液体被过冷, 液体的结构平衡时间 $\tau_{\mathrm{eq}}$ 在接近玻璃化转变温度 $T_{\mathrm{g}}$ 附近时迅速增加, 使得液体在温度降到 $T_{\mathrm{g}}$ 以下时从动力学角度看束 缚在非平衡的玻璃态中. 当在 $T_{\mathrm{g}}$ 以下退火时, 这个玻璃态逐渐弛豫到平衡态. 越来越多的证据表明这个弛豫的过程非常复杂, 而且受到势 能景图的影响. 本文通过研究不同的小分子玻璃形成系统发现从玻璃转变到过冷液体的过程中出现了一个分层的老化路径. 通过差示扫 描量热仪实验发现在 $T_{\mathrm{g}}$ 以下退火时, 玻璃态先进入到一个暂时的亚稳态过冷液体. 在 $T_{\mathrm{g}}$ 以下, 这个液体的焓值稍微比平衡态过冷液体高. 通过拉曼散射测量结构的实验同样证实了这个发现. 这个暂时的亚稳态到稳态过冷液体的转变动力学在温度 $T_{\mathrm{sp}}$ 处表现出了热力学转变的 特征, 并且导致了一系列反常现象比如在脆性指数图上出现的类相变行为. 这些发现暗示了过冷液体的自由能景图在接近 $T_{\mathrm{g}}$ 的时候变得 复杂而且具有质的变化, 同时对玻璃化转变过程有很明显的影响. 